NASA/TM-1999-209047

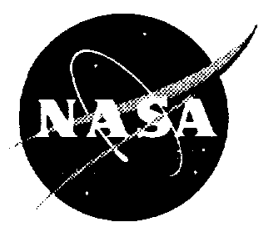

\title{
Space Acceleration Measurement System-II: Microgravity Instrumentation for the International Space Station Research Community
}

Thomas J. Sutliff

Glenn Research Center, Cleveland, Ohio

Prepared for the

16th Instrumentation and Measurement Technology Conference sponsored by the Institute of Electrical and Electronics Engineers Venice, Italy, May 24-26, 1999

National Aeronautics and Space Administration

Glenn Research Center 
This report is a preprint of a paper intended for presentation at a conference. Because of changes that may be made before formal publication, this preprint is made available with the understanding that it will not be cited or reproduced without the permission of the author.

Available from

NASA Center for Aerospace Information 7121 Standard Drive

Hanover, MD 21076

Price Code: A03
National Technical Information Service 5285 Port Royal Road Springfield, VA 22100

Price Code: A03 


\title{
Space Acceleration Measurement System-II: Microgravity Instrumentation for the International Space Station Research Community
}

\author{
Thomas J. Sutliff \\ National Aeronautics and Space Administration \\ Glenn Research Center \\ Cleveland, Ohio 44135, U.S.A.
}

\begin{abstract}
The Intemational Space Station opens for business in the year 2000, and with the opening, science investigations will take advantage of the unique conditions it provides as an on-orbit laboratory for research. With initiation of scientific studies comes a need to understand the environment present during research. The Space Acceleration Measurement System-ll provides researchers a consistent means to understand the vibratory conditions present during experimentation on the International Space Station.

The Space Acceleration Measurement System-II, or SAMS-II, detects vibrations present while the space station is operating. SAMS-II on-orbit hardware is comprised of two basic building block elements: a centralized control unit and multiple Remote Triaxial Sensors deployed to measure the acceleration environment at the point of scientific research, generally within a research rack. Ground Operations Equipment is deploved to complete the command. control and data telemetry elements of the SAMS-II implementation.

Initially, operations consist of user requirements development, measurement sensor deployment and use, and data recovery on the ground. Future system enhancements will provide additional user functionality and support more simultaneous users.
\end{abstract}

\section{Introduction}

The International Space Station (ISS) provides a research platform in space to conduct investigations that are just not possible on earth. The space station provides an environment known as microgravitya, allowing basic and applied research in numerous disciplines to take place. These science disciplines span the areas of biotechnology, combustion, fluids science, fundamental physics, and materials science.

a microgravity: the condition resulting from free-fall of an orbiting body: this condition provides an apparent weightless state to an item relative to its surroundings
To ensure valid research it is necessary to measure the parameters of the experiment. Much like one measures temperature when conducting a thermal experiment, the acceleration variable needs to be measured while conducting microgravity experiments. Vibrations exist on the space station for a variety of reasons: equipment operation, structural motion, crew movement, and thermal expansion are but a few. Table 1 lists some typical events and their effective frequency and amplitude ranges[1]. Residual steady-state accelerations are present due to atmospheric drag,

\begin{tabular}{|c|c|c|}
\hline Event & $\begin{array}{l}\text { Frequency } \\
\text { Range (Hz) }\end{array}$ & $\begin{array}{l}\text { Amplitude } \\
\text { Range }(\mu g)\end{array}$ \\
\hline Reboost, thrusters & $0-100+$ & $\begin{array}{c}300- \\
30,000\end{array}$ \\
\hline Crew Exercise & $0-5$ & $50-1.000$ \\
\hline $\begin{array}{l}\text { Equipment Operation } \\
\text { (Shuttle data) }\end{array}$ & $1-150$ & $10-500$ \\
\hline Structural system & $0.04-25+$ & $0.1-100$ \\
\hline Atmospheric Drag & $0-0.01$ & $0.1-1.0$ \\
\hline Rotational Effects & $0-0.1$ & $0.01-1$ \\
\hline Vehicle Maneuvers & $0-10+$ & $1-100$ \\
\hline
\end{tabular}

Table 1. Typical Acceleration Events

rotational accelerations, and gravity gradient effects. No matter how one designs a space station, some residual vibration and quasi-steady acceleration will always be present. The presence of this environment may not be a problem, but when scientists try to study theoretical effects of removing gravity through on-orbit experiments, the effects of acceleration tend to confuse their experimental results unless the actual environment is accounted for. The SAMS-II project is responsible for measuring the vibratory accelerations for all U.S. microgravity science payloads who may require them. The SAMS-II instrument directly supports the science disciplines contained within the Microgravity Research Program of the National Aeronautics and Space Administration's Office of Life and Microgravity Science and Applications by providing a consistent means of measuring the residual vibration environment during microgravity research.

SAMS-II provides a service to investigators who need measurements taken- collecting the data, ensuring its validity, and providing basic analysis 
services. Additional capability for data reduction and analysis is available from the Principal Investigator Microgravity Services (PIMS) project, described later, which is responsible for understanding the scientific relevance of the collected data, conducting further analyses on the data, and providing counseling to individual investigators who might be unfamiliar with on-orbit acceleration measurement characteristics and their effects on an experiment.

\section{Requirements}

The researchers within the science disciplines that are conducting initial investigations aboard the International Space Station have a difficult job- they are designing experiments to be conducted aboard a vehicle which itself is being designed and built. SAMS-II has asked these same researchers to define their requirements for the acceleration measurement system that will support them. In conjunction with the inputs from users of similar instruments, a generalized system was defined that supports the entire set of space station microgravity researchers, meeting their initial measurement needs as well as those expected as the space station becomes operational and research expertise develops.

The legitimacy of these requirements was driven home in 1992, when the U.S. National Academy of Sciences/ National Research Council stated that the microgravity environment must be measured to quantify it for the research being conducted. In a document discussing the future of microgravity research, the National Research Council stated the following challenge:

"The $g$-level must be measured accurately, locally, frequently, and synchronously with every experiment." [2]

A measurement system to address this challenge was devised for science investigations on the space shuttle. The Space Acceleration Measurement System (SAMS) has flown over 20 times since early 1991 [3]. Now a new system, SAMS-II, is in final stages of development with its first deployment expected aboard the International Space Station in the year 2000. Similarities between the shuttle-based SAMS hardware and the space-station bound SAMS-II units exist, but significant technological improvements in hardware, such as high performance analog-to-digital converters enable enhancements in performance to be made in this second generation measurement system. The space station capabilities also provide an ability for instruments to function in a more lab-like setting. The SAMS-II takes advantage of these improvements within its design implementation [4]. Furthermore, the SAMS-II is designed to accommodate an ongoing upgrade program by leveraging continued technology enhancements in hardware capabilities.

To state the requirements simply, the SAMS-II must measure the acceleration environment for multiple payloads simultaneously conducting research in space throughout the lifetime of the International Space Station. It must accurately acquire this data, do it within the available resources and the constraints of the space station. It must then provide this information to the end user, the scientist, in various formats, all within a timely manner.

\section{Capabilities of SAMS-II}

SAMS-II has a distributed architecture design, which results in a measurement system that is expandable, upgradable, and deployable aboard the International Space Station. Multiple Remote Triaxial Sensor (RTS) systems are able to be deployed near any payload requiring direct measurements of the acceleration environment. A controller, initially consisting of a laptop computer, uses the space station Medium Rate Link (MRL) network to connect the independent RTS systems together on-orbit and provides a single point communication link to the SAMS-II Ground Operations Equipment at a Telescience Support Center (TSC) where data are received for distribution to users. Figure 1 schematically shows the system's data flow.

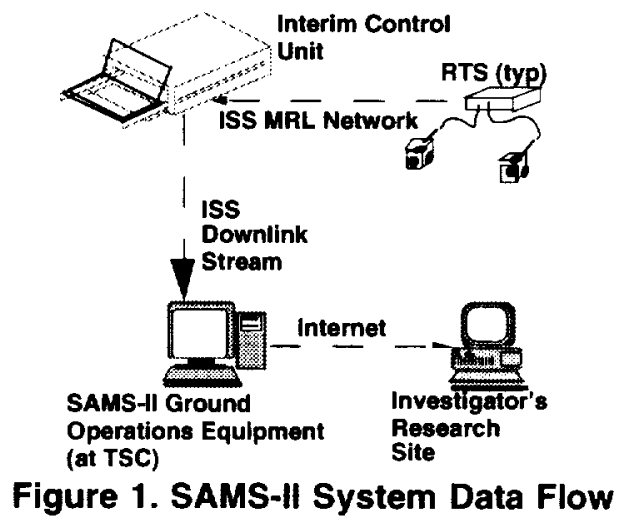

SAMS-II relies on the infrastructure of the space station similar to the manner in which a researcher relies on the capabilities of his laboratory to conduct science investigations. In this manner, duplication of resources is avoided, and the needs of users are met while minimizing the complexity and cost of developing the SAMS-II. The ISS MRL serves as the conduit to move data from source to receiver. The distributed RTS units support multiple users while offering individual control over the collected data. Users are able to modify data acquisition parameters in a manner as if they "owned" the hardware by selecting the frequency range measured by the RTS, 
thereby minimizing the load on network Iraffic. Onboard signal conditioning and conversion into engineering units are implemented to provide a direct feedback to payloads and crew of the current environment, if desired. A centralized controller system provides a means to keep track of acquired data and each unit's health. Modular software and hardware allow for simplified enhancements and upgrades to the systems.

While the space station is still under construction, a subset of the ultimate vehicle capability is being provided to allow initiation of research. The early deployment of an Interim Control Unit (ICU) follows suit, providing a limited number of initial users a set of core measurement services. Data conversion, collection, and dissemination to the ground are being provided. Ultimately, the ICU will be upgraded to a full-fledged Control Unit. This upgrade enables onboard data analysis and direct feedback of information to payloads and the crew. This upgrade also enables on-board control of experimental parameters with the hope of optimizing the experiment operations. The Control Unit deployment is timed to coincide with initial operations of the major research facilities being developed in biotechnology, combustion, fluid physics, materials science, and low temperature physics. As these facilities are deployed, it is expected that more simultaneous measurements will be required and additional SAMS-II Control Unit performance features will be desired.

Throughout this deployment of interim and final control capability, the core element of SAMS-II is the Remote Triaxial Sensor system. This system consists of two sensor heads and a supporting electronics unit, which are installed in multiple locations throughout the space station. Each RTS is capable of providing measurements from $0.01 \mathrm{~Hz}$ up beyond $300 \mathrm{~Hz}$. This wide-band region is known as the vibratory, or g-jitter regime. Amplitudes are expected from 1 micro-g up to as high as 10 milli-g. The RTS heads are capable of measuring across this range and beyond, up to $0.1 \mathrm{~g}$, should there be a higher amplitude transient disturbance taking place.

\section{Interim Control Unit Capabilities}

The SAMS-II controller unit being deployed for the initial operations and research stage is known as the Interim Control Unit, and provides basic command and control features for the limited number of distributed RTS units present during early utilization. The ICU relies on the availability of existing laptop computers which are flight ruggedized and deployed by the space station program.
Functionally, the ICU provides a single location at which the software required to operate an RTS is housed. Whenever an RTS is powered up, the ICU recognizes the RTS, instructs it across the space station network to configure itself according to preprogrammed settings and allows the RTS to initiate measurements for the payload customer. A time synchronization is performed bringing RTS time in line with ICU time. After the data from the RTS is received by the ICU. it is checked for completeness, combined with data from other sensors and hardware performance data, and downlinked to the ground.

The ICU computer subsystem is a ThinkPad laptop from the ISS pool of flight grade Portable Computer Systems. To communicate with the RTS systems, the ICU software operates across the space station payload Ethernet network known as the Medium Rate Link in a two-way communication mode similar to that used to network computers together hetween offices. This laptop is integrated into an International Subrack Interface Standard (ISIS) drawer along with ICU power and cooling subsystems. Although not expected to be needed for baseline operation, the ICU laptop has sufficient hard disk capacity to store up to 10 hours of data from five sensor heads running at their maximum frequency range. This capability is provided in case downlink services are interrupted, and it allows onorbit research to continue until services are restored.

\section{Remote Triaxial Sensor Capabilities}

The SAMS-II Remote Triaxial Sensor systems consist of two primary building block elements: the RTS-Sensor Enclosure (SE) and RTS-Electronics Enclosure (EE). [4] Each element serves a distinct role in the measurement of microgravity acceleration aboard the International Space Station. An RTS-EE and two RTS-SE units are shown in Figure 2 along with one RTS interface cable.

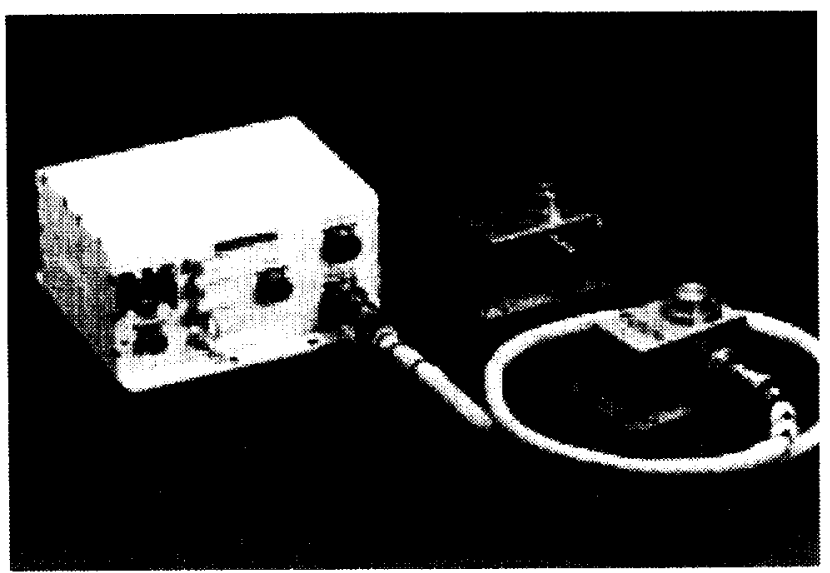

Figure 2. Remote Triaxial Sensor System 
Each Sensor Enclosure is mounted as close as possible to the experiment being supported to best measure the environment present at the point of research. The SE contains the primary transducer elements which sense the vibratory excitation, signal conditioning, and 24 bit analog-to-digital converters. The vibration transducers are commercially available single axis, servo-driven pendulous mass accelerometers typically used for inertial guidance and navigation purposes. The A-to-D converters are programmable, providing versatility in selecting sample rate/acquisition frequency ranges of interest based on research requirements and are located within the SE to minimize the influence of measurement noise. The dynamic range of the 24 bit converters in each SE is sufficiently large to eliminate the need for gain-changing circuitry during on-orbit operations. Integrated temperature transducers in the accelerometers also allow for temperature compensation of the acceleration data when the acceleration data are applied against temperature calibration curves. Precise orthogonal alignment to better than $0.1^{\prime \prime}$ between the three sensors provides a triaxial measurement of the environment. The calibration of each SE is based on the use of scale factor, hias, and temperature compensation coefficients independently established on the ground for each accelerometer. These values are provided within a look-up table for each axis of an SE. A recall of the on-orbit SE units for recalibration is planned to occur nominally every two years. Units are expected to maintain stability through this period, as they are not installed in harsh vibratory environments.

Each Electronics Enclosure provides power and command signals for up to two Sensor Enclosures and receives the temperature and digital acceleration data from each SE. The EE executes compensation for temperature and residual alignment effects, and optionally converts the data to engineering units. The Electronics Enclosure contains network interface circuitry to provide connectivity across the space station Ethernet via standard internet protocols to the centralized SAMS-II controller.

Generally, the EE hardware is embedded within a science or facility rack at the time of its initial launch, and an RTS cable is routed to a more accessible Sensor Enclosure which is likely to be installed onto a particular science payload when the payload is brought up on orbit. Variations on installation methods for both the EE and the SE are negotiated by SAMS-II with each end user. An example of an RTS-EE installation is shown in Figure 3, where an EE (labeled "SAMS-II Electronics Enclosure") is embedded within the midshelf of an EXPRESS (EXpedite the PRocessing of Experiments to Space Station) Rack and two SE connection points ("SAMS Sensor Ports") are brought to the front panel for easy middeck locker payload accessibility.

NASA/TM-1999-209047

\section{Ground Operations Equipment}

A third element within the SAMS-II architecture does not fly aboard space station, yet it is as critical to the successful completion of the mission of acquiring and disseminating acceleration data to the research community as the on-orbit hardware. This element is the Ground Operations Equipment (GOE). The SAMSIl GOE is located in the Telescience Support Center (TSC) within the NASA Glenn Research Center. The TSC provides users an entry to the command, control, and data dissemination that occur between the space station and the SAMS-II GOE. The TSC is also implementing its ISS capabilities in phases, due to the progressive implementation of on-ground research capabilities.

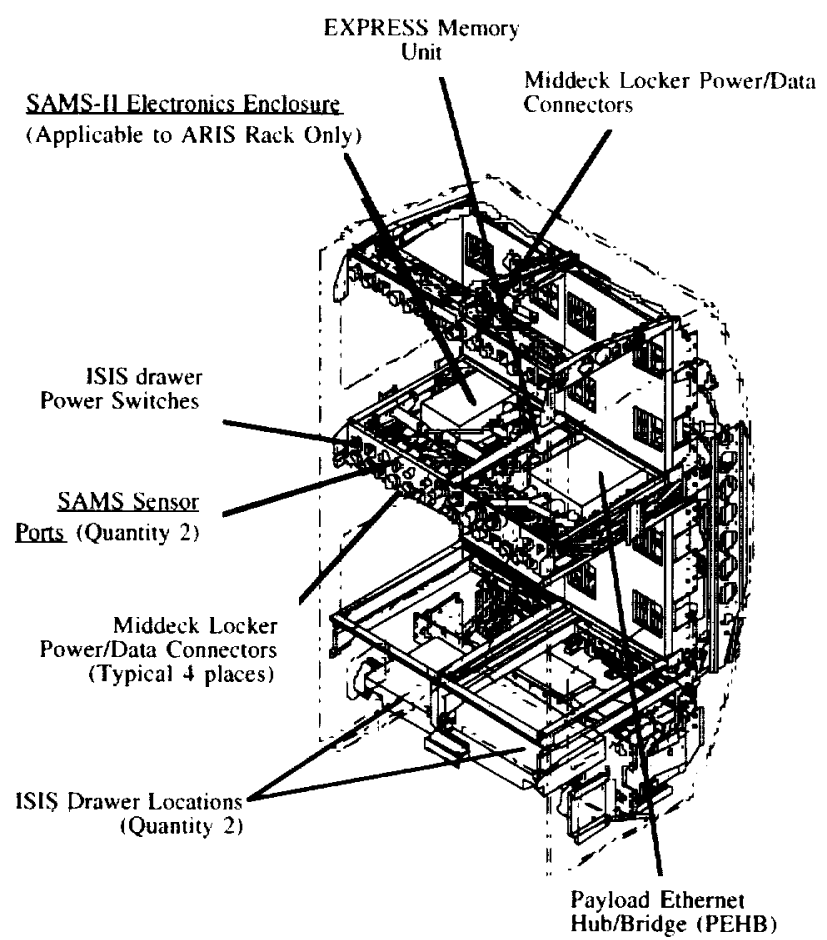

Figure 3. RTS-EE within EXPRESS Rack

The Ground Operations Equipment performs two key functions. It maintains control, monitoring the health, status, and performance of the operational onorbit elements of SAMS-II, and it provides a means by which the volume of downlinked data can be provided to the principal investigator (PI) conducting research. These data are also provided to the PI Microgravity Services (PIMS) organization for analysis and characterization of the environment to provide PI's with information about the microgravity environment. The PIMS team has conducted analysis of SAMS and OARE (a quasi-steady acceleration sensor instrument) data from the early 1990's and have established userpreferred data formats for numerous shuttle missions. 
The PIMS team is responsible for making the data available to the end user in as expeditious a manner as possible.

During early utilization, the GOE is the mechanism by which changes in SAMS-II on-orbit configurations are made. These changes are implemented via commands issued by ground operators. At the transition from ICU to CU operations, commanding from remote sites will be implemented. Remote commanding will allow autonomy for a PI, providing the investigator direct control of the performance configuration of their sensor.

\section{Performance Validation}

As SAMS-II reaches the end of its development cycle, it is necessary to verify the system capabilities versus the stated requirements. The deployed system must meet a multitude of environmental requirements, such as exposure to $3 \mathrm{~g}$ to $4 \mathrm{~g}$ launch vibration accelerations, withstanding exposure to electromagnetic interference, and meeting other various crew and vehicle imposed requirements. These verifications are conducted at both subsystem and system levels. Verification methods in use to demonstrate the design has met requirements include inspections, analyses, and tests. A final validation of performance is also required. Validation for SAMS-II is complicated by the phasing of deployment stages by the ISS, TSC, and the SAMS-II controller. And since not all the SAMS-II hardware with true ISS interfaces will be available at any one point in time, conducting a final end-to-end system test must wait until SAMS-II arrives on-orbit. To minimize the risk imposed by these constraints, simulators are invoked at various stages to accomplish the verifications and system demonstrations in a modular fashion at the subsystem levels for the RTS and the ICU.

\section{On-Orbit Operations}

Following delivery of the first on-orbit hardware, a short period of system checkout and an integrated validation test is conducted. This point is the first time in which all flight hardware operational elements (SAMS-II, EXPRESS Rack, and ISS network) are integrated.

After system checkout, operations begin and include payload-specific Principal Investigator support and involve acquiring long streams of contiguous data from as many sensors as are deployed. The newness of the vehicle (ISS) and this measurement system, in combination, provide a unique opportunity to gain an understanding of the current vehicle performance to meet its requirement to provide conditions for its payloads. Such insight may allow future operational changes, expanding the research capability of the ISS.
Operations commence on ISS flight 6A, currently scheduled for launch in April, 2000. For initial utilization, SAMS-II deploys the ICU within an ISIS drawer on EXPRESS Rack \#2, an Active Rack Isolation System (ARIS)-equipped rack. The Physics of Colloids in Space fluids experiment [4] is the first science user supported with SAMS-II measurements, and coincidentally is housed within the same EXPRESS rack. This sub-rack experiment is supported by an SE connected to the embedded EE. Two additional RTS-EE units are housed within two ISIS drawers in EXPRESS Rack \#1, supporting three SE rack-interface measurements being taken around the boundary of EXPRESS Rack \#2 to perform measurements of the input environment to the ARIS.

The ICU, RTS-EE's and RTS-SE's are monitored on the GOE at the Telescience Support Center; any outof-tolerance voltages, temperatures or processor conditions are detected and displayed on the GOE. Warning and shutdown alarms are detected within the GOE, with a subset of shutdown conditions being provided to the EXPRESS Rack for on-orbit detection. General status of resources, such as data throughput, on-board storage available, and RTS configuration logging is also conducted by the SAMS-II GOE.

The collected data are downlinked to the GOE. Concurrently with being checked for obvious erroneous results, the data are routed to the Principal Investigator Microgravity Services (PIMS) group for data processing. As the data are received, the PIMS analysis routines are applied, converting the acceleration-versus-time records into power spectral density (PSD) records. Each PSD is accumulated over time and a color spectrogram of the acceleration versus frequency versus time is generated [1]. This spectrogram, along with other real-time calculated parameters such as peak and root-mean-square values over a set time period are made available across a world-wide-web accessible link. SAMS-IL/PIMS expects that updates will be made available at 2 minute intervals.

PIMS also conducts a general analysis of all the acquired data, and provides characterization assessments of the environment so that future researchers and payload developers may benefit from knowledge of the general environment of this new research facility.

\section{Customers}

A variety of groups with differing perspectives constitute the customer base for SAMS-II. Specific Principal Investigators and their science teams as well as the payload developer teams all require support from the SAMS-II and PIMS teams to deliver acceleration measurement data from the ISS environment. 
Principal Investigators - The PI is provided with experiment specific measurements. Measurements must be acquired near the science payload, as structural transmissibility characteristics alter the environment to which the scientific experiment is exposed. There is more variation in transmissibility the further the measurement is taken from the experiment location. In addition, PIMS works with the PI for a particular experiment to determine any specialized data analysis needs. As part of this educational effort, PIMS annually offers a Microgravity Environment Interpretation Tutorial.

Payload developers - Each experiment is developed by a payload developer. The SAMS-II project works with these developers to ensure proper integration of the RTS hardware. Payload specific interface agreements are developed and negotiated between SAMS-II and each payload to define installation configurations and streamline the integration process. Payloads include sub-rack size units and rack-level facilities. Besides the Physics of Colloids in Space [5] experiment, SAMS-II supports and is on schedule to provide measurement services to the Microgravity Research Program for on-orbit deployments by the following disciplines and facilities:

a) Biotechnology (BioTechnology Facility, May 2004)

b) Combustion (Combustion Integrated Rack. October 2002)

c) Fluids Science (Fluids Integrated Rack, June 2003)

d) Fundamental Physics (Low Temperature Microgravity Physics Facility, April 2004)

e) Materials Science (Material Science Research Facility- three racks, October 2002, June 2003. and July 2004)

In addition, the multi-user EXPRESS Rack program will receive four RTS-EE units (deployed April and August 2000, January and July 2004). The embedded EE subsystem becomes a service available to users and, in combination with a SE provided to the subrack payload, provides the payload a full RTS measurement capability.

The multi-user Microgravity Science Glovebox also has been allocated an RTS system for use as early as August 2000.

\section{Future Enhancements}

SAMS-II continues to evolve with the ongoing utilization of the International Space Station. Not only are there ongoing preventative and remedial maintenance plans, but as technology advances, it is anticipated that upgrades to both the hardware and performance will be possible.
As stated earlier, one planned upgrade is the transition from the Interim Control Unit to a more capable Control Unit. The ICU will be replaced by the Control Unit, which is currently scheduled to be put into operation by 2002. The Control Unit provides capability for acquisition of more sensors, and a capability to conduct processing and data analysis onboard the International Space Station. The Control Unit also accepts the quasi-steady acceleration data from the Microgravity Acceleration Measurement System data stream [6]. Further data processing and distribution back to on-orbit user facilities and payloads is possible in addition to nominal data transmission to the ground.

\section{Conclusion}

SAMS-II has been developed to provide a consistent means of providing vibratory acceleration measurements to the microgravity research community aboard the International Space Station. The system measures across the expected magnitude and frequency ranges of the vibration environment over which the space station researchers have concern. The results of the measurements provided by SAMS-II are necessary to understand the interdependence between the environment and experimental results. This understanding is needed for the development of theoretical models and the documentation of results in fundamental scientific studies.

\section{References}

[1] DeLombard. R.. Compendium of Information for Interpreting the Microgravity Environment of the Orbiter Spacecraft, NASA TM-107032, 1996

[2] Toward a Microgravity Research Strategy. Space Studies Board. National Research Council, 1992.

[3] DeLombard. R.. Finley. B. D., et. al., Development and Flight Results from the Space Acceleration Measurement System (SAMS), NASA TM-105625 (AIAA 92-0345), 1992.

[4] Sutliff, T. J., Requirements and Development of an Acceleration Measurement System for International Space Station Microgravity Science Payloads. NASA TM-107484, 1998.

[5] Ansari. R. R.. Hovenac E. A., et. al., Physics of Colloids in Space Experiment. Space Technology and Applications International Forum- STAIF.99, 1999.

[6] Rice, J. E., Fox, J. C.. et. al., Microgravity Acceleration Measurement System for the International Space Station, IEEE IMTC/99 \#9089, 1999. 

Public reponting burden for this collection of intormation is estimated to average 1 hour per response, including the time for reviewing instructions. searching existing data sources gathering and maintaining the data needed, and completing and reviewing the collection of iniormation. Send comments regarding this burden estimate or any other aspect of this collection of information, including suggestions for reducing this burden, to Washington Headquarters Services. Directorate tor information Operations and Reports, 1215 Jefterson Davis Highway. Suite 1204. Artington. VA 22202-4302, and to the Office of Management and Budget, Paperwork Reduction Project (0704-0188), Washington. DC 20503.
1. AGENCY USE ONLY (Leave blank)
2. REPORT DATE
March 1999
3. REPORT TYPE AND DATES COVERED
Technical Memorandum

4. TITLE AND SUBTITLE

Space Acceleration Measurement System-II: Microgravity Instrumentation for the International Space Station Research Community

\section{AUTHOR(S)}

Thomas J. Sutliff

\section{PERFORMING ORGANIZATION NAME(S) AND ADDRESS(ES)}

National Aeronautics and Space Administration

John H. Glenn Research Center at Lewis Field

Cleveland, Ohio 44135-3191

5. FUNDING NUMBERS

WU-398-95-0A-00

\section{SPONSORINGMONITORING AGENCY NAME(S) AND ADDRESS(ES)}

National Aeronautics and Space Administration

Washington, DC 20546-()(0)1

\section{PERFORMING ORGANIZATION} REPORT NUMBER

$\mathrm{E}-11578$

\section{SUPPLEMENTARY NOTES}

Prepared for the 16th Instrumentation and Measurement Technology Conference, sponsored by the institute of Electrical and Electronics Engineers, Venice, Italy, May 24-26, 1999. Responsible person, Thomas J. Sutliff, organization code 6727, (216) 433-3887.

\section{2a. DISTRIBUTIONAVAILABILITY STATEMENT}

12b. DISTRIBUTION CODE

Unclassified - Unlimited

Subject Categories: $17,19,32,33$, and 62

Distribution: Nonstandard

This publication is available from the NASA Center for AeroSpace Information, (301) 621-0390.

\section{ABSTRACT (Maximum 200 words)}

The International Space Station opens for business in the year 2000, and with the opening, science investigations will take advantage of the unique conditions it provides as an on-orbit laboratory for research. With initiation of scientific studies comes a need to understand the environment present during research. The Space Acceleration Measurement System-Il provides researchers a consistent means to understand the vibratory conditions present during experimentation on the International Space Station. The Space Acceleration Measurement System-II, or SAMS-II, detects vibrations present while the space station is operating. SAMS-II on-orbit hardware is comprised of two basic building block elements: a centralized control unit and multiple Remote Triaxial Sensors deployed to measure the acceleration environment at the point of scientific research, generally within a research rack. Ground Operations Equipment is deployed to complete the command, control and data telemetry elements of the SAMS-II implementation. Initially, operations consist of user requirements development, measurement sensor deployment and use, and data recovery on the ground. Future system enhancements will provide additional user functionality and support more simultaneous users.

\section{SUBJECT TERMS}

Acceleration; Microgravity; International Space Station; Instrumentation; Measurement

17. SECURITY CLASSIFICATION
OF REPORT
Unclassified

18. SECURITY CLASSIFICATION
OF THIS PAGE
Unclassified
19. SECUAITY CLASSIFICATION OF ABSTRACT Unclassified

NSN 7540-01-280-5500 